\title{
RESPUESTA HIDROLÓGICA Y EROSIVA DE UN SUELO FORESTAL MEDITERRÁNEO EN RECUPERACIÓN DE DIFERENTES IMPACTOS
}

\author{
V. Andreu, e. Gimeno-García, O. González-Pelayo, J. Campo \& J. L. Rubio
}

Centro de Investigaciones sobre Desertificación-CIDE (CSIC, Univ. de Valencia, Generalitat

Valenciana). Camí de la Marjal, s/n.46470 - Albal (España).

E-mail.: Vicente.andreu-perez@uv.es

\begin{abstract}
Land use changes, including land abandonment, and forest fires have been two key factors that have characterized the evolution of Mediterranean ecosystems. This work studies the evolution in the hydrologic characteristics and the erosive response of a Mediterranean forest soil, which has undergone during years changes in its forest use and the fire impact, are shown. This soil is in recovering stage since year 1996. The study has been carried out in the Experimental Station of Porta-Coeli in a system of four erosion plots $(40 \times 8 \mathrm{~m})$ of closed type. The hydrologic behaviour and erosive parameters have been studied in each rain event during period 2006-2008. The temporal variability of soil moisture contents have been also studied through the characterization of its response curves regarding different episodes of rain, analyzing its relation with runoff generation.
\end{abstract}

Keywords: Hydrology dynamics; Erosion processes; Plots; Soil moisture content; Forest fire; Runoff; Rain regime.

RESUMEN.- En las últimas décadas, dos de los factores más importantes que han caracterizado la evolución en los ecosistemas mediterráneos han sido los cambios de uso del suelo, incluyendo el abandono de cultivos, y los incendios forestales. En este trabajo se presenta la evolución de las características hidrológicas y de la respuesta erosiva de un suelo forestal mediterráneo que ha sufrido durante años cambios en su uso y el impacto de incendios forestales. Actualmente la zona de estudio se encuentra en recuperación desde el año 1996. El trabajo se ha desarrollado en la Estación Experimental de Porta-Coeli en un sistema de cuatro parcelas (40 $\times 8 \mathrm{~m})$ de erosión de tipo cerrado. Se ha determinado su comportamiento hidrológico y sus parámetros erosivos en cada episodio de lluvia durante el período 2006-2008. También se ha estudiado la variabilidad temporal del contenido de humedad del suelo 
a través de la caracterización de sus curvas-respuesta en diferentes episodios de lluvia, y se analiza su relación con la generación de escorrentía.

Palabras clave: Dinámica hidrológica; procesos de erosión; parcelas; humedad del suelo; incendio forestal; escorrentía; lluvias.

\section{Introduction}

Los ecosistemas mediterráneos se caracterizan por sostener una compleja y rica biodiversidad, tanto en fauna como en flora, y una elevada edafodiversidad, pero también por soportar una larga historia de presión humana. No obstante, estos ecosistemas, en gran parte, se mantienen sobre un frágil equilibrio basado en el difícil mantenimiento de sus condiciones hidrológicas. Bajo un clima cambiante que evoluciona hacia condiciones más duras, con incremento en la temperatura media, mayor frecuencia en la incidencia de sequías, y disminución en las precipitaciones pero con aumento del número de episodios agresivos (CHRISTENSEN et al., 2007; IPCC, 2007), junto con una creciente presión humana y sus implicaciones en la intensificación de los procesos de degradación ambiental, la sostenibilidad de este equilibrio se hace cada vez más compleja.

Una de las amenazas ambiéntales más importantes identificadas por la Unión Europea es la erosión del suelo, que afecta con mayor o menor intensidad a un cuarto de su superficie. Se ha observado que en los últimos años, las actividades humanas han producido un incremento acelerado de este proceso, debiéndose principalmente a la degradación o desaparición de la cubierta vegetal (EEA, 2005).

En este sentido, la evolución de los procesos de erosión en el área mediterránea se basa principalmente en dos fenómenos con una fuerte componente socio-económica, e imbricados entre sí: los cambios en los usos del suelo y los incendios forestales. Los primeros se han ido intensificando desde la segunda mitad del siglo XX, debido tanto a la migración progresiva de la población rural hacia los núcleos urbanos o de producción, como a los cambios en la política agrícola de la Unión Europea. En ambos casos se ha favorecido el abandono de zonas agrícolas poco productivas o marginales, lo que ha dado lugar a importantes transformaciones en el paisaje, incrementando las cubiertas naturales de bosque y matorral (LASANTAMARTÍNEZ et al., 2005). Este fenómeno ha conducido también a cambios en la hidrología de laderas favoreciendo en muchos casos una reducción en los procesos erosivos (BEGUERÍA et al., 2006; BOIX-FAYOS, 2008). No obstante, 
en las zonas áridas y semi-áridas mediterráneas la recolonización vegetal se ralentiza debido a las duras condiciones climáticas y al déficit hídrico que incide en gran parte del año. Con ello, el suelo queda sin una cubierta vegetal protectora efectiva contra la agresividad de la lluvia, lo que conlleva consecuencias importantes en los procesos de erosión y sedimentación (FAULKNER et al., 2003; GOVERS et al., 2006), sobre todo en los primeros años después del abandono (PARDINI et al., 2003).

En muchos casos, esta colonización vegetal favorece el aumento de la biomasa, que en períodos secos incrementaría el riesgo de incidencia de los incendios forestales (PARDINI et al., 2004; LLOVET et al., 2009). Estos constituyen una de las mayores amenazas para los bosques europeos meridionales, afectando como media cada año a 500.000 ha a través de la incidencia de 50.000 incendios (EUROPEAN COMMISSION, 2003).

El efecto del fuego se traduce en cambios en las características físicas, químicas e hidrológicas de los suelos, y en la degradación de la cubierta vegetal, que en muchos casos implica el aumento de procesos de erosión acelerada (RUBIO et al., 1997; PARDINI et al., 2004). En general, las causas de este incremento post-incendio de los procesos erosivos se han atribuido al desarrollo de hidrofobicidad superficial (PROSSER \& WILLIAMS, 1998; DOERR et al., 2006), a la destrucción de la cubierta protectora del suelo (JOHANSEN et al., 2001; PIERSON et al., 2008), a los cambios en la estabilidad de los agregados (ANDREU et al., 2001; SHAKESBY \& DOERR, 2006), a la alteración del contenido en materia orgánica del suelo (SHAKESBY \& DOERR, 2006) y al efecto de la capa superficial de cenizas.

El impacto de los incendios dependerá tanto de las características intrínsecas del fuego como de las características propias de la zona afectada (climatología, tipo de suelo, cubierta vegetal, pendiente, morfología y del contenido en humedad de la biomasa superficial), y de su severidad. Todo ello influirá no sólo el grado de agresión sufrida por el ecosistema sino también su capacidad de recuperación, su tiempo de resiliencia.

El objetivo del presente trabajo ha sido el estudio de la evolución de las características hidrológicas y de la respuesta erosiva de un suelo forestal mediterráneo, que ha soportado durante años cambios en su uso y el impacto de incendios forestales. Este suelo se encuentra en recuperación desde el año 1996. Para ello se ha utilizado un sistema de parcelas de erosión de tipo cerrado incluido en la Estación Experimental de Porta-Coeli (Valencia). Los parámetros climáticos y edáficos se han monitorizado de forma constante en cada uno de los episodios de lluvia registrados durante el período 2006-2008.

Por otro lado, una forma de obtener información más precisa sobre el proceso de generación de escorrentía es la obtención de datos continuos sobre la evolución del contenido de humedad del suelo y relacionar esta 
información con la respuesta de producción de escorrentía bien a la escala de las parcelas experimentales o a la escala de cuenca. Las medidas temporales de la humedad del suelo pueden ser utilizadas para identificar umbrales críticos de humedad por encima de los cuales se puede generar escorrentía superficial, dependiendo, además, de las características intrínsecas de los episodios de lluvia. Así mismo, se han caracterizado las curvas de respuesta de la humedad del suelo frente a diferentes episodios de lluvia y se han analizado sus relaciones con la generación de escorrentía a escala de parcela.

\section{Metodología}

\section{1. Área de estudio}

El trabajo se ha desarrollado en la Estación Experimental de Porta-Coeli (término municipal de Serra, provincia de Valencia). Está situada en la vertiente oriental de la Sierra Calderona (Figura 1). Sus coordenadas geográficas son $7^{16} 43^{9}$. Es un área que en su historia refleja la dinámica típica de presión humana y usos que caracteriza a amplias zonas forestales de la cuenca mediterránea, así como de la incidencia de incendios forestales, de los que se tiene referencia desde 1905 y de los que el último tuvo lugar en 1978.

La Estación ocupa una ladera forestal ligeramente cóncava, con orientación Sur-Oeste, y con una pendiente media del 20\%. La litología de la zona corresponde a materiales triásicos del Muschelkalk constituidos por margas arcillo-areniscosas y conglomerados. Existen también afloramientos de material fuertemente consolidado y de naturaleza conglomerática, en estratos perpendiculares a la pendiente, que ocupan entre un $8-10 \%$ de la superficie de las distintas parcelas. La fácil alterabilidad y meteorización del material de origen y la presencia en toda la zona de afloramientos del material originario da lugar a una elevada pedregosidad, tanto en el interior del perfil como en superficie.

Los suelos, de escaso espesor, pertenecen al tipo Leptosol rendzínico (F.A.O.-UNESCO, 1988). En la Tabla 1 se expone la descripción de las características macro-morfológicas de un perfil del suelo representativo de la zona. Este suelo se caracteriza por presentar una textura franco-arcilloarenosa, una elevada estabilidad estructural y buena capacidad de retención de agua (Tabla 2). 


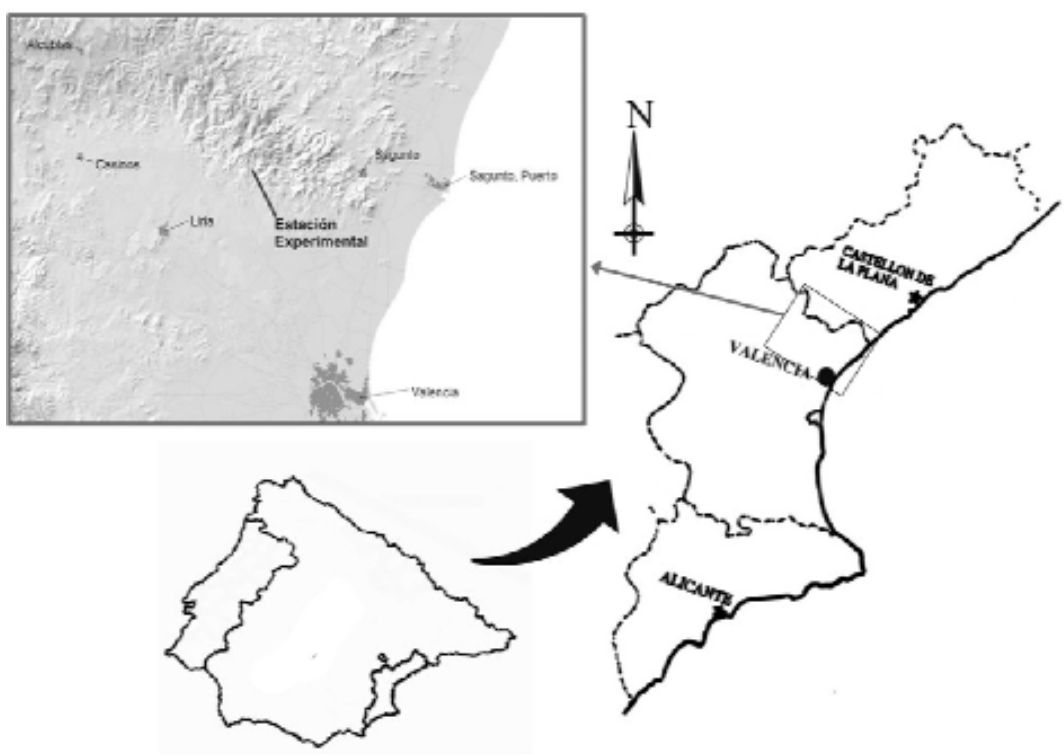

Figura 1. Localización del área de estudio.

Figure 1. Location of the study area.

Tabla 1. Descripción del perfil de suelo típico del área de la Estación Experimental de Porta Coeli (Valencia).

Table 1. Profile description of the characteristic soil of the Porta-Coeli Expeimental Station (Valencia).

\begin{tabular}{lll}
\hline Profundidad & Horizonte & Descripción \\
\hline $0-18$ & Ah & Pardo amarillento oscuro en seco (19YR 4/4) y pardo amarillento
\end{tabular}
oscuro en húmedo (10YR 3/4); franco arcillo arenoso; estructura moderada, subangular fina a granular gruesa; ligeramente adherente, ligeramente plástico, friable en húmedo y blando en seco; pocos poros y canales, continuos, inped, simples y transversales y frecuentes poros, discontinuos, caóticos, exped; frecuentes gravas y pocas piedras, angulares, redondeadas y planas, no meteorizadas, de caliza; calcáreo; comunes raíces, medianas, finas y muy finas; transición gradual y ondulada a un AC.

18-32 AC Pardo rojizo en húmedo (5YR 4/3); franco arcillo arenoso; estructura moderada, subangular mediana; ligeramente adherente, ligeramente plástico, friable en húmedo; pocos poros, finos y muy finos, discontinuos, caóticos, inped y tubulares; frecuentes gravas y piedras, angulares, no meteorizadas; calcáreo; pocas raíces; transición brusca e irregular al $\mathrm{R}$.

$+32 \quad \mathrm{R} \quad$ Calizas del Muschelkalk


Tabla 2. Algunas de las características físicas y químicas más importantes del suelo de PortaCoeli.

Table 2. Some physical and chemical characteristics of the Porta-Coeli soil.

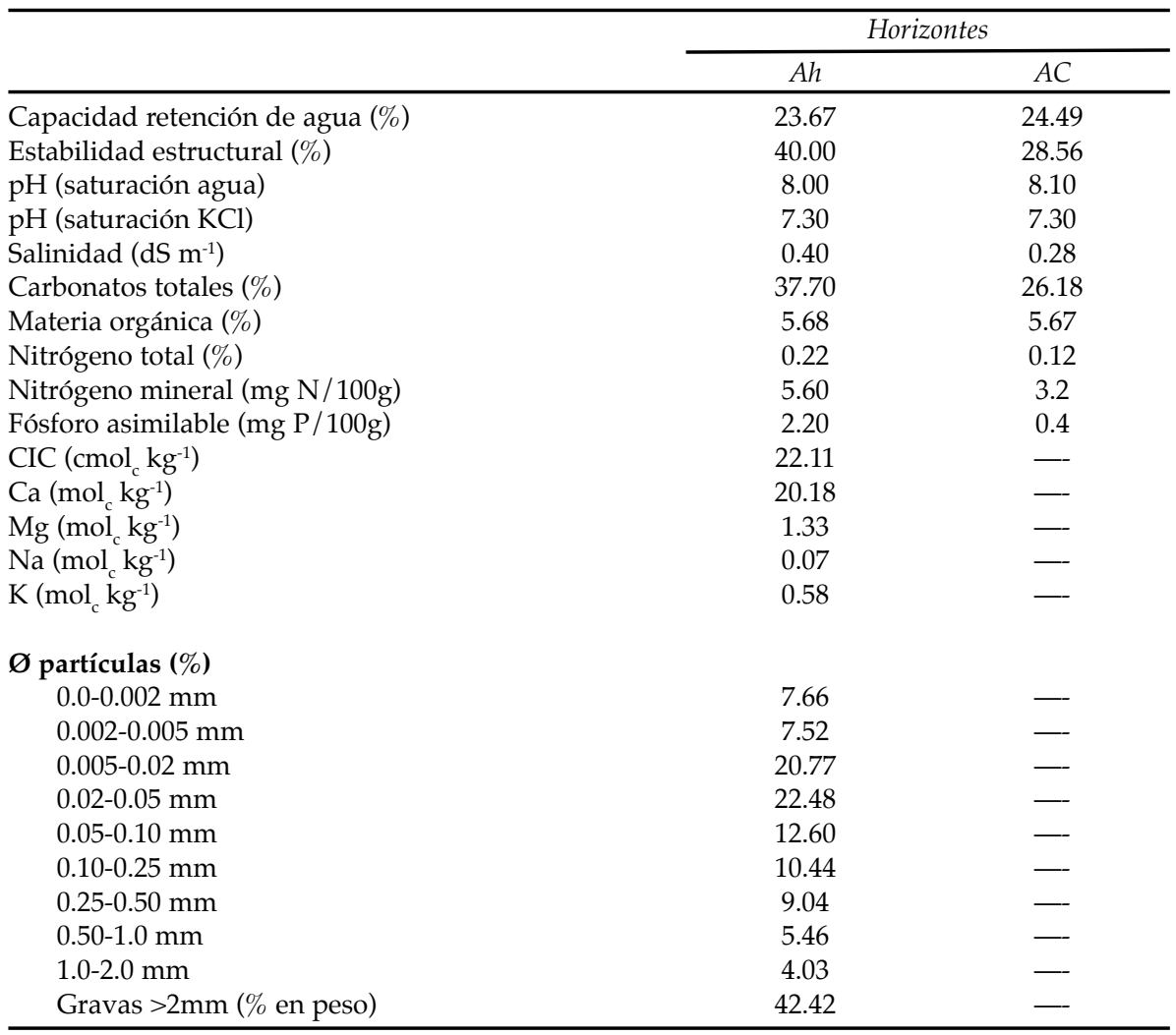

La clasificación climática según el método de Thornthwaite indica que el tipo climático para esta estación es seco subhúmedo $\left(C_{1}\right)$, con moderado exceso de agua en invierno (s), mesotérmico (valores altos de eficacia térmica $\mathrm{B}_{2}{ }_{2}$ ) y con valores bajos de eficacia térmica estival. Según la clasificación bioclimática de RIVAS-MARTÍNEZ (1981 y 1987), pertenece al piso Termomediterráneo (temperatura media anual $15.7^{\circ} \mathrm{C}$ ) y presenta un ombroclima seco (precipitación media anual $572 \mathrm{~mm}$ ).

En cuanto a la vegetación, el tipo dominante pertenece a la alianza Rosmarino-Ericion, siendo las especies más representativas Rosmarinus officinalis, Erica multiflora, Stipa tenacissima, Chamaerops humilis y Thymus vulgaris. Su cobertura en las distintas parcelas varía entre un 50 y un $60 \%$. 


\subsection{Diseño experimental}

La instalación consiste en cuatro parcelas experimentales de erosión de $320 \mathrm{~m}^{2}(40 \times 8 \mathrm{~m})$ cada una, todas ellas aisladas en su perímetro por ladrillos de $40 \mathrm{~cm}$ de altura (bardos) cubiertos de barniz hidrofóbico, y orientadas en sentido de la pendiente. Entorno al límite superior de las parcelas se realizó un canal de drenaje, de $40 \mathrm{~cm}$ de profundidad, para interrumpir los flujos de escorrentía superficial y subsuperficial procedentes de la parte más alta de la ladera. La zona terminal inferior se estrecha hasta alcanzar una anchura de 2 $\mathrm{m}$ para lograr la confluencia de flujos de escorrentía, acabando en un sistema cerrado de colectores metálicos de $2 \mathrm{~m}$ de frente que a su vez van conectados a depósitos de $2000 \mathrm{~L}$ de capacidad para la recogida global tanto de escorrentía como de sedimentos. En el interior de cada uno de estos depósitos existe otro de $40 \mathrm{~L}$ de capacidad que recibe directamente el flujo de los colectores, su cometido es concentrar los sedimentos arrastrados para facilitar su recogida y posterior análisis.

En 1987 se estableció su diseño original, que perseguía el estudio comparativo de la eficacia de diferentes especies arbustivas con características forrajeras para el control de la erosión. Una de las parcelas conservó la vegetación natural (T1). En otra de ellas se plantaron 90 unidades de Medicago arbórea en grupos de tres individuos con una separación de $20 \mathrm{~cm}$ entre ellos, formando setos transversales dispuestos al tresbolillo para frenar la escorrentía y los arrastres de suelo (T2). De la misma forma a la anterior, se plantaron en otra de las parcelas 90 individuos de Atriplex nummularia, que fueron cambiados en 1990 por Psoralea bituminosa al no dar una buena respuesta en su crecimiento (T3). Por último, se mantuvo una parcela desprovista de vegetación, lo que se realizó en primer lugar por métodos manuales y posteriormente se mantuvo desnuda por tratamientos esporádicos con herbicidas (Herbión MCPA® y ROUNDOUP®). Esta parcela se constituyó para ser utilizada como control (T4).

En 1996 se seleccionaron dos de las cuatro parcelas de la Estación para realizar quemas experimentales simulando diferentes intensidades de fuego, la parcela que presentaba la vegetación natural (T1), que se había regenerado tras sufrir un incendio en 1978, y la parcela que fue usada para la plantación de Psoralea bituminosa (T3) y que en ese momento presentaba una escasa cobertura vegetal $(\leq 22 \%)$. Se alcanzaron temperaturas medias entre $127^{\circ} \mathrm{C}$ y $385{ }^{\circ} \mathrm{C}$ en la superficie del suelo considerando que los fuegos fueron de moderada a baja intensidad, aunque los mayores tiempos de permanencia de las temperaturas fueron en T1. A partir del verano de 1996 se dejó de actuar en las parcelas quedando en regeneración desde entonces, en las mismas condiciones. 


\subsection{Determinaciones y análisis}

Para cuantificar el desarrollo y características de cada uno de los episodios de lluvia de la zona, y para posibilitar la obtención de estos datos en tiempo real, la Estación consta de un sistema de monitorización y toma de datos por sensores y transmisión de estos a distancia vía GSM. Este sistema proporciona además datos de temperatura y humedad tanto ambientales como del suelo de forma continua, así como de la velocidad y dirección del viento. Las características de cada episodio de lluvia (duración, evolución, volumen de lluvia) se registran por medio de un sensor pluviométrico que toma medidas cada 2 minutos realizando la media cada 10. La intensidad de la lluvia se calcula como $\mathrm{I}_{30}$ en base a los datos anteriores.

Las medidas temporales del contenido de humedad del suelo se han realizado mediante una sonda reflectómetro CS616, que se encuentra instalada vertical y permanentemente a una profundidad de $10 \mathrm{~cm}$, en las proximidades de la estación meteorológica. Se almacenan los datos mínimos, máximos y medios diarios de humedad del suelo, así como los datos diarios en intervalos de 1 hora. Estos datos se expresan en porcentaje de contenido volumétrico de humedad.

En cada episodio de lluvia se midieron tanto la escorrentía producida como el sedimento generado en cada una de las parcelas. El caudal sólido se muestrea sobre la base de los sedimentos arrastrados y / o en suspensión en el agua de escorrentía, producida en cada episodio de lluvia. Se debe tener en cuenta que la cantidad recogida en cada depósito es el total generado por la parcela correspondiente. La escorrentía y los sedimentos se recogen inmediatamente después de finalizada la lluvia. Se han considerado como episodios de lluvia erosivos a aquellos que al menos hayan presentado generación de escorrentía.

Para el estudio de la caracterización de los tipos de curvas de respuesta de humedad del suelo se han analizado los datos diarios en intervalos de 1 hora correspondientes a 41 episodios de lluvia registrados durante los años 2006, 2007 y 2008. La selección de los episodios de lluvia se ha basado aquellos que han producido un pico de humedad del suelo y/o se ha registrado escorrentía en los depósitos de las cuatro parcelas. La amplitud de los intervalos de tiempo analizados es muy variable, y está en función de la frecuencia de los episodios de lluvia y de la respuesta del suelo. Para cada uno de los episodios seleccionados se han considerado las variables: (1) humedad del suelo justo antes del inicio de la precipitación $\left(\mathrm{HS}_{a n t}\right) ;(2)$ humedad máxima del suelo durante la precipitación $\left(\mathrm{HS}_{\max }\right)$, y (3) la relación entre ambas variables $\left(\mathrm{HS}_{a n t} / \mathrm{HS}_{\max }\right)$. El estudio de las relaciones entre estas 
variables y la producción de escorrentía se ha realizado en 23 episodios seleccionados de todo el conjunto inicial, que se corresponden a lluvias individuales, en las que no ha habido ni precipitación ni escorrentía acumulada de episodios próximos.

\subsection{Análisis de los datos}

Los datos analizados son los correspondientes a las variables relacionadas con la lluvia (volumen total, $\mathrm{I}_{30}$, duración), la hidrología (producción de escorrentía, tasa de infiltración, coeficiente de escorrentía, $\mathrm{HS}_{a n t^{\prime}} \mathrm{HS}_{\max }, \mathrm{HS}_{a n t}$ / $\mathrm{HS}_{\max }$ ) y de pérdida de suelo (sedimento generado, arrastre de sedimento). Se ha realizado el ANOVA para identificar la existencia de diferencias entre las distintas parcelas para las variables seleccionadas. En los casos en los que no se ha podido asumir la normalidad de la distribución ni la homogeneidad de la varianza, se han empleado los tests no paramétricos de Kruskal-Wallis y Mann-Whitney $(\mathrm{P} \leq 0.05)$. Se han realizado las correlaciones rho de Spearman para el estudio de las relaciones entre las variables seleccionadas. También se ha realizado el análisis de los modelos lineales por pasos sucesivos y diferentes estimaciones de ajuste de modelos no lineales, para confirmar cuales son las variables de mayor peso en relación tanto a la generación de escorrentía como a la pérdida de suelo.

\section{Resultados y Discusión}

\subsection{Características de las precipitaciones}

Dentro de la variabilidad característica del clima mediterráneo, con incidencia casi cíclica de períodos de sequía o con escasez de precipitaciones, el período 2006-2008 podría considerarse como la fase de atenuación de un período seco, que se inició en 2004 (Figura 2) y que alcanzó su mínimo en 2006 ( $323.20 \mathrm{~mm}$ de lluvia total). El valor de 2006 puede considerarse como el mínimo de la década. No obstante, durante el período estudiado se ha observado un cambio en cuanto a la distribución típica de las lluvias en ambientes mediterráneos, distribuyéndose de forma más homogénea a lo largo del año e incidiendo también en el período estival. 


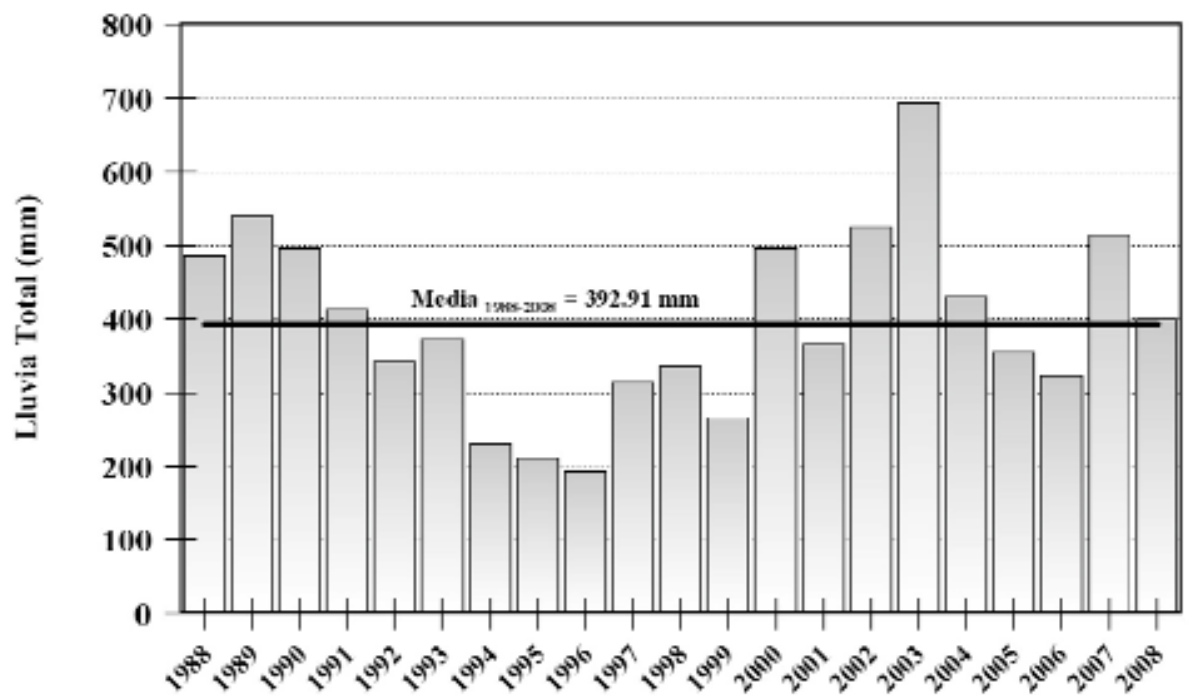

Figura 2. Precipitaciones totales anuales registradas en la Estación Experimental de Porta-Coeli durante el período 1988-2008, con su precipitación media global (línea).

Figure 2. Total anual rais occurred in the Porta-Coeli Experimental Station during 1988-2008, with its global average (line).

Durante los tres años estudiados (2006-2008) se han registrado en la zona de la Estación Experimental 321 episodios de lluvia con un volumen total de $1326.20 \mathrm{~mm}$. Entre estos tres años la variabilidad ha sido sustancial tanto en el volumen total de las precipitaciones como en sus características, no existiendo correspondencia alguna entre los tres años en el volumen caído, la duración de los episodios o su intensidad. Así, el 2007 fue el año con mayor precipitación $(514.3 \mathrm{~mm})$ pero con menor $\mathrm{I}_{30}$ media $\left(8.23 \mathrm{~mm} \mathrm{~h}^{-1}\right)$, mientras que el 2006 presentó la menor precipitación $(323.20 \mathrm{~mm})$ pero la mayor $\mathrm{I}_{30}$ media $\left(10.2 \mathrm{~mm} \mathrm{~h}^{-1}\right)$. El 2008 presentó valores ligeramente superiores al 2006

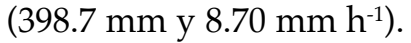

Estas diferencias también se han reflejado en el número de lluvias erosivas, es decir, que presentaron una generación de escorrentía medible. De los 321 episodios de lluvia ocurridos sólo en 37 se observó generación de escorrentía (11.50\%), y de estos en 7 se midió producción de sedimento, lo que representa que sólo un $2.20 \%$ de las lluvias produjeron arrastre de suelo. En la Figura 3 se pueden apreciar las características de los 37 episodios de lluvia erosiva ocurridos en la Estación Experimental. En general las precipitaciones no alcanzaron intensidades muy elevadas, con un máximo en todo el período 
de $34 \mathrm{~mm} \mathrm{~h}^{-1}(17 / 5 / 08)$. Un $70.27 \%$ de los episodios presentó intensidades inferiores a $10 \mathrm{~mm} \mathrm{~h}^{-1}$, que junto con un aumento progresivo y general en su duración (Figura 3) indica un carácter poco agresivo. El volumen medio de los episodios erosivos han sido de $19.23 \mathrm{~mm}$, con un valor máximo de 80.80 $\mathrm{mm}\left(\mathrm{I}_{30}=15.60 \mathrm{~mm} \mathrm{~h}^{-1} ; 26 / 1 / 07\right)$. En general, aproximadamente el $60 \%$ de las precipitaciones fueron de menos de $20 \mathrm{~mm}$, concentrándose 4 de los 5 episodios de más de $40 \mathrm{~mm}$ en 2007. Sin embargo, las lluvias en ese año presentaron las media más baja de intensidad $\left(8.23 \mathrm{~mm} \mathrm{~h}^{-1}\right)$.

\subsection{Respuesta hidrológica}

Durante el período estudiado, los 37 episodios de lluvia erosivos ocurridos dieron lugar a $27.37 \mathrm{~mm}$ (8725.09 L) de escorrentía en la Estación Experimental. Reflejándose el comportamiento general de las precipitaciones en sus características. Así, la escorrentía generada en 2006 y 2008 fue un $70.38 \%$ y $43.95 \%$ menor, respectivamente, a la de 2007. Del análisis del total de estos valores no se han observado diferencias significativas entre años en este parámetro ni en el coeficiente de escorrentía, pero si en cuanto a la tasa de infiltración, que fue mucho menor durante 2008 (media de $1.38 \mathrm{~mm} \mathrm{~h}^{-1}$ ).

Entre los distintos tratamientos, es el T4 (parcela que se mantuvo sin vegetación hasta 1996) el que ofrece los mayores valores de escorrentía en todo el período (Tabla 3), seguido del T1 (parcela que sufrió un incendio en 1996). El comportamiento de ambos tratamientos se va distanciando cada vez más del que presenta el T2 (parcela de antigua plantación de Medicago arbórea), mientras que el T3 (parcela de antigua plantación de Atriplex nummularia) queda en un nivel intermedio pero más cercano a T1. No obstante, se ha determinado, que es a partir de lluvias de una intensidad mayor a $15 \mathrm{~mm} \mathrm{~h}^{-1}$ cuando se dan los mayores valores de generación de escorrentía, casi igualándose el comportamiento de T1 y T4, y de T2 y T3, aumentando la diferencia entre ambos bloques. Los valores observados en los distintos tratamientos son netamente inferiores a los observados por ANDREU et al. (1998) previamente a las quemas experimentales, en el período (1988-1993). El impacto del fuego se hace evidente en el cambio de comportamiento del tratamiento T1, que antes del fuego presentaba siempre los valores más bajos de escorrentía frente a los demás tratamientos y en la actualidad se asimila a los valores de T4, tratamiento que siempre ha dado los mayores valores. En todos los casos, los valores de generación de escorrentía son menores a los encontrados en la bibliografía consultada. En particular, los valores determinados en T2 y T3 son menores a los cuantificados para parcelas de erosión, terrazas y campos abandonados por JOEL et al. (2002), 
KOULOURI \& GIOURGA (2007), BAKKER et al. (2008), respectivamente, en ambientes mediterráneos. O incluso mayores a los observados para T4, como son los registrados en estudios sobre suelos devegetados por herbicidas y diversas prácticas agrícolas realizados por MICKELSON et al. (2001).

Tabla 3. Valores de las características hidrológicas y de pérdida de suelo en los distintos tratamientos, durante el período 2006-2009.

Table 3. Values of the main hidrologic and soil loss parameters in the different treatments, during 20062008.

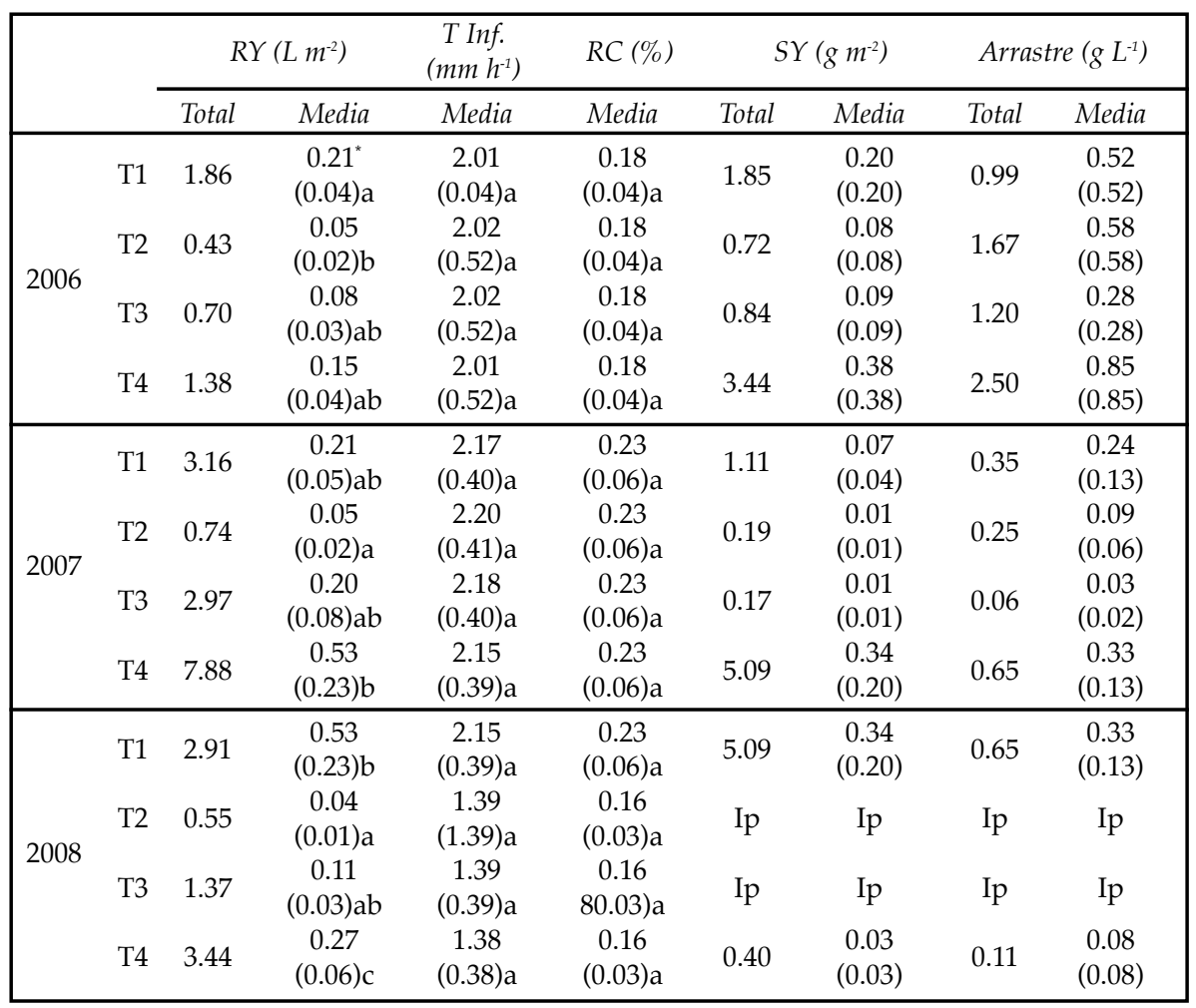

$\mathrm{RY}=$ Volumen de Escorrentía; $\mathrm{T}$ Inf.: Tasa de infiltración; $\mathrm{RC}=$ Coeficiente de Escorrentía; $\mathrm{SY}$ : Sedimento.

(*) Valores medios con distinta letra indican diferencias significativas.

$(\mathrm{P} \leq 0.05)$ Error típico entre paréntesis. 
Los valores obtenidos, sobre todo en T1, han sido también inferiores, en general, a los reflejados por distintos estudios sobre el efecto de los incendios forestales en condiciones ambientales similares en sistemas de parcelas (CAMPO et al., 2006; LLOVET et al., 2009).

El umbral medio de $\mathrm{I}_{30}$ que genera escorrentía en el período de estudio se ha mostrado igual para T1, T3 y T4, siendo de $1.4 \mathrm{~mm} \mathrm{~h}^{-1}$, mientras que en el T2 fue de $3.8 \mathrm{~mm} \mathrm{~h}^{-1}$. Todo esto indica unas mejores condiciones hidrológicas en el tratamiento $\mathrm{T} 2$ respecto a los demás, sobre todo a T1 y T4, que son los que sufrieron impactos de mayor entidad. Esto se ve confirmado también en los valores de la tasa de infiltración y de coeficiente de escorrentía (Tabla 3). En general, las tasas de infiltración y los coeficientes de escorrentía son muy similares entre los distintos tratamientos e indican un comportamiento hidrológico favorable en la mayoría de los casos. De esta forma, el valor medio de coeficiente de escorrentía para el período estudiado fue de 0.19 , que es un valor menor al máximo del rango establecido para este tipo de suelos forestales (0.25) (CORBITT, 1999; CHIN, 2006). Hay que considerar también el efecto que ha tenido la distribución más uniforme de las precipitaciones a lo largo del año y la mayor duración de estas.

De los análisis estadísticos realizados se ha confirmado el comportamiento particular del año 2008 frente a 2006 y 2007, sobre todo en la tasa de infiltración. Entre tratamientos, las diferencias más significativas se han observado en la generación de escorrentía (Tabla 3). Así, existen diferencias significativas entre el T2 y T4 tanto en 2007 como en 2008, y entre T2 y T1 en 2006. Esto podría indicar una cierta mejora en las condiciones de T1 frente a $\mathrm{T} 4$, respecto a los valores menores que se observaron en T2.

Respecto a los factores que más influyen en la respuesta hidrológica (Tablas 4 y 5) también se observan diferencias. En general, los factores que tienen más fuerza en cuanto a la generación de escorrentía son la $I_{30}$ y el volumen de lluvia, para los tratamientos T1 a T3. Sin embargo, T4 (suelo que se mantuvo sin cobertura hasta el año 1996) presenta como factor principal al volumen de precipitación y como secundarios al coeficiente de escorrentía y a la tasa de infiltración, mientras que la intensidad no parece influir (Tabla 5). Esto podría indicar un estado de mayor fragilidad en el caso de T4 al ser más sensible a cambios en la cantidad de precipitación independientemente de su agresividad. Del análisis de modelos de regresión múltiple en pasos sucesivos (Tabla 5), se observa también que T2 presentaría las mejores condiciones hidrológicas ya que el factor principal en este caso sería la intensidad de la lluvia, es decir su agresividad antes que su cantidad. Durante el período 19881993 (ANDREU et al., 1998) para los tratamientos T3 y T4 aparecía la duración del episodio de lluvia como efecto de mayor importancia después del volumen de lluvia, en lugar de la $\mathrm{I}_{30}$. Esto puede indicar una mejora en las condiciones generales de todos los tratamientos, pero sobre todo en T3 y T4. 
V. ANDREU, E. GIMENO-GARCÍA, O. GONZÁLEZ-PELAYO, J. CAMPO \& J. L. RUBIO

Tabla 4. Coeficientes de correlación Rho de Spearman para las variables estudiadas (G: Análisis global de los datos. Tx: Análisis por tratamientos).

Table 4. Rho Spearman correlation coefficients for the studied variables (G: Analysis of the global data set. Tx: Analysis per treatments).

\begin{tabular}{|c|c|c|c|c|c|c|c|c|}
\hline & & Duración & V & $I_{30}$ & $R Y$ & T Inf. & $R C$ & $S Y$ \\
\hline $\mathrm{RY}$ & $\begin{array}{l}\mathrm{G} \\
\mathrm{T} 1 \\
\mathrm{~T} 2 \\
\mathrm{~T} 3 \\
\mathrm{~T} 4\end{array}$ & $\begin{array}{c}0.284^{* *} \\
0.373^{*} \\
\text { n.s. } \\
\text { n.s. } \\
0.462^{* *}\end{array}$ & $\begin{array}{l}0.540^{\star *} \\
0.674^{\star *} \\
0.526^{\text {** }} \\
0.593^{\star *} \\
0.715^{\text {** }}\end{array}$ & $\begin{array}{l}0.504^{\star *} \\
0.616^{\text {** }} \\
0.618^{\text {** }} \\
0.525^{\text {** }} \\
0.634^{\text {** }}\end{array}$ & & & & \\
\hline T Inf. & $\begin{array}{l}\mathrm{G} \\
\mathrm{T} 1 \\
\mathrm{~T} 2 \\
\mathrm{~T} 3 \\
\mathrm{~T} 4 \\
\end{array}$ & $\begin{array}{c}-0.392^{* *} \\
-0.390^{*} \\
-0.393^{*} \\
-0.392^{*} \\
-0.395^{*}\end{array}$ & $\begin{array}{l}0.435^{* *} \\
0.437^{\star *} \\
0.434^{\star *} \\
0.435^{* *} \\
0.433^{\star *}\end{array}$ & $\begin{array}{l}0.632^{\text {** }} \\
0.630^{\text {** }} \\
0.634^{* *} \\
0.633^{* *} \\
0.631^{* *}\end{array}$ & $\begin{array}{c}0.296^{\star *} \\
0.359^{\star} \\
0.515^{\star *} \\
0.385^{*} \\
\text { n.s. }\end{array}$ & - & & \\
\hline R. Coef. & $\begin{array}{l}\mathrm{G} \\
\mathrm{T} 1 \\
\mathrm{~T} 2 \\
\mathrm{~T} 3 \\
\mathrm{~T} 4\end{array}$ & $\begin{array}{l}0.595^{\star *} \\
0.596^{* \star} \\
0.594^{* *} \\
0.596^{* *} \\
0.597^{* *}\end{array}$ & $\begin{array}{l}0.999^{* *} \\
1.000^{* *} \\
1.000^{* *} \\
1.000^{* *} \\
1.000^{\star *}\end{array}$ & $\begin{array}{l}0.683^{* *} \\
0.679^{* *} \\
0.686^{* *} \\
0.683^{* *} \\
0.679^{* *}\end{array}$ & $\begin{array}{l}0.521^{\text {** }} \\
0.665^{\text {** }} \\
0.524^{\text {** }} \\
0.590^{\star *} \\
0.710^{\text {** }}\end{array}$ & $\begin{array}{l}0.432^{* *} \\
0.432^{* *} \\
0.434^{* *} \\
0.431^{* *} \\
0.426^{* *}\end{array}$ & - & \\
\hline SY & $\begin{array}{l}\text { G } \\
\text { T1 } \\
\text { T2 } \\
\text { T3 } \\
\text { T4 }\end{array}$ & $\begin{array}{l}\text { n.s. } \\
\text { n.s. } \\
\text { n.s. } \\
\text { n.s. } \\
\text { n.s. }\end{array}$ & $\begin{array}{c}0.168^{*} \\
\text { n.s. } \\
\text { n.s. } \\
\text { n.s. } \\
\text { n.s. }\end{array}$ & $\begin{array}{c}0.370^{* *} \\
0.410^{*} \\
0.353^{*} \\
0.353^{*} \\
0.420^{\star *}\end{array}$ & $\begin{array}{c}0.436^{* *} \\
0.439^{* *} \\
0.355^{\star} \\
0.392^{*} \\
0.484^{* *}\end{array}$ & $\begin{array}{c}0.433^{* *} \\
0.519^{* *} \\
0.370^{*} \\
0.370^{*} \\
0.511^{\text {* }}\end{array}$ & $\begin{array}{l}\text { n.s. } \\
\text { n.s. } \\
\text { n.s. } \\
\text { n.s. } \\
\text { n.s. }\end{array}$ & \\
\hline Arrastre & $\begin{array}{l}\mathrm{G} \\
\mathrm{T} 1 \\
\mathrm{~T} 2 \\
\mathrm{~T} 3 \\
\mathrm{~T} 4\end{array}$ & $\begin{array}{c}-0.162^{*} \\
\text { n.s. } \\
\text { n.s. } \\
\text { n.s. } \\
\text { n.s. }\end{array}$ & $\begin{array}{l}\text { n.s. } \\
\text { n.s. } \\
\text { n.s. } \\
\text { n.s. } \\
\text { n.s. }\end{array}$ & $\begin{array}{c}0.363^{* *} \\
0.381^{*} \\
0.348^{*} \\
0.348^{*} \\
0.388^{*}\end{array}$ & $\begin{array}{c}0.426^{* \star} \\
0.409^{\star} \\
0.347^{\star} \\
0.388^{*} \\
0.453^{\star *}\end{array}$ & $\begin{array}{c}0.429^{* *} \\
0.494^{* *} \\
0.371^{*} \\
0.371^{*} \\
0.489^{* *}\end{array}$ & $\begin{array}{l}\text { n.s. } \\
\text { n.s. } \\
\text { n.s. } \\
\text { n.s. } \\
\text { n.s. }\end{array}$ & $\begin{array}{l}0.998^{* *} \\
0.993^{* *} \\
0.999^{* *} \\
0.999^{* *} \\
0.990^{* *}\end{array}$ \\
\hline
\end{tabular}

V: Volumen de lluvia; $I_{30}$ : Intensidad de la lluvia en 30 minutos; RY: Producción de escorrentía (L $\left.\mathrm{m}^{-2}\right)$; T Inf.: Tasa de infiltración ( $\left.\mathrm{mm} \mathrm{h}^{-1}\right)$; RC: Coeficiente de Escorrentía (\%); SY: Producción de sedimento $\left(\mathrm{g} \mathrm{m}^{-2}\right)$.

**, * La correlación es significativa al nivel 0.01 y 0.05 , respectivamente. n.s.: correlación no significativa. 
RESPUESTA HIDROLÓGICA Y EROSIVA DE UN SUELO FORESTAL MEDITERRÁNEO...

Tabla 5. Regresión multiple por pasos sucesivos entre los diferentes parámetros estudiados $(\mathrm{Y}=$ $\left.\mathrm{B}_{0}+\mathrm{B}_{1} \mathrm{X}_{1}+\mathrm{B}_{2} \mathrm{X}_{2}+\ldots ..\right)$.

Table 5. Multiple step-wise regressions between the studied parameters $\left(Y=B_{0}+B_{1} X_{1}+B_{2} X_{2}+\ldots ..\right)$.

\begin{tabular}{|lcccccc|}
\hline Tratamiento & $Y$ & $B_{0}$ & $B_{j}$ & $X_{j}$ & $R^{2}$ & "Sig. \\
\hline T1 & Escorrentía & 0.040 & $\mathrm{~B}_{1}=0.592$ & $\mathrm{X}_{1}=\mathrm{V}$ & 0.608 & 0.025 \\
& & & $\mathrm{~B}_{2}=0.291$ & $\mathrm{X}_{2}=\mathrm{I}_{30}$ & & \\
& Sedimento & -0.084 & $\mathrm{~B}_{1}=0.434$ & $\mathrm{X}_{1}=\mathrm{I}_{30}$ & 0.188 & 0.007 \\
& Arrastre & -0.171 & $\mathrm{~B}_{1}=0.399$ & $\mathrm{X}_{1}=\mathrm{I}_{30}$ & 0.159 & 0.014 \\
\hline T2 & Escorrentía & -0.006 & $\mathrm{~B}_{1}=0.437$ & $\mathrm{X}_{1}=\mathrm{I}_{30}$ & 0.491 & 0.013 \\
& & & $\mathrm{~B}_{3}=0.371$ & $\mathrm{X}_{3}=\mathrm{V}$ & & \\
& Sedimento & -0.017 & $\mathrm{~B}_{1}=0.405$ & $\mathrm{X}_{1}=\mathrm{I}_{30}$ & 0.164 & 0.013 \\
& Arrastre & -0.227 & $\mathrm{~B}_{1}=0.377$ & $\mathrm{X}_{1}=\mathrm{I}_{30}$ & 0.142 & 0.021 \\
\hline T3 & Escorrentía & -0.045 & $\mathrm{~B}_{1}=0.788$ & $\mathrm{X}_{1}=\mathrm{V}$ & 0.621 & 0.000 \\
& Sedimento & -0.039 & $\mathrm{~B}_{1}=0.385$ & $\mathrm{X}_{1}=\mathrm{I}_{30}$ & 0.148 & 0.019 \\
& Arrastre & -0.105 & $\mathrm{~B}_{1}=0.360$ & $\mathrm{X}_{1}=\mathrm{I}_{30}$ & 0.130 & 0.028 \\
\hline T4 & Escorrentía & 0.15 & $\mathrm{~B}_{1}=29.585$ & $\mathrm{X}_{1}=\mathrm{V}$ & 0.999 & 0.008 \\
& & & $\mathrm{~B}_{2}=-28.756$ & $\mathrm{X}_{2}=\mathrm{RC}$ & & \\
& & & $\mathrm{B}_{3}=-0.021$ & $\mathrm{X}_{3}=\mathrm{T}$ Inf. & & \\
& Sedimento & -0.253 & $\mathrm{~B}_{1}=0.519$ & $\mathrm{X}_{1}=\mathrm{RY}$ & 0.495 & 0.042 \\
& & & $\mathrm{~B}_{2}=0.291$ & $\mathrm{X}_{2}=\mathrm{I}_{30}$ & & \\
& Arrastre & -0.338 & $\mathrm{~B}_{1}=0.442$ & $\mathrm{X}_{1}=\mathrm{I}_{30}$ & 0.195 & 0.006 \\
\hline
\end{tabular}

(") Significación a $\mathrm{p}<0.05, \mathrm{n}=37 ; \mathrm{V}=$ Volumen de lluvia; $\mathrm{t}=$ Duración de la lluvia; $\mathrm{I}_{30}=$ Intensidad de la lluvia en 30'; RY= Volumen de Escorrentía; $\mathrm{RC}=$ Coeficiente de Escorrentía; $\mathrm{T}$ Inf.: Tasa de infiltración.

Del estudio del ajuste a diferentes modelos se ha observado que en todos los casos la generación de escorrentía se representa mejor en un modelo lineal frente al volumen de lluvia (Figura 4), sobre todo en T4. En los demás tratamientos, también podría ajustarse positivamente a un modelo cuadrático.

\subsection{Curvas de respuesta de la humedad del suelo}

Del análisis de los datos continuos de humedad del suelo en los episodios de lluvia seleccionados, se ha observado la existencia de tres comportamientos diferentes durante el periodo de estudio en la estación Experimental de Porta-Coeli (Figura 5). El análisis estadístico confirma las diferencias entre los tres tipos de comportamiento observados (Tabla 6). Del conjunto de variables empleadas, el volumen de precipitación, la humedad 
Vol. Lluvia

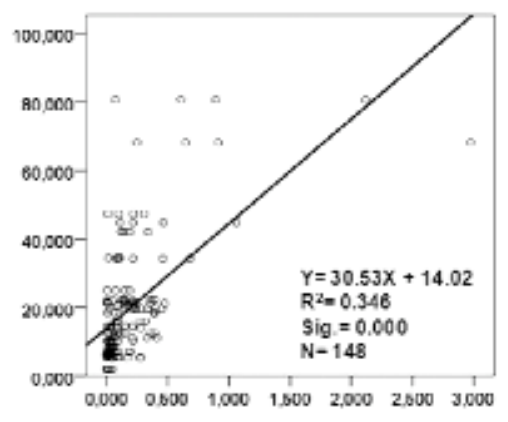

Escorrentia
$\mathbf{I}_{30}$

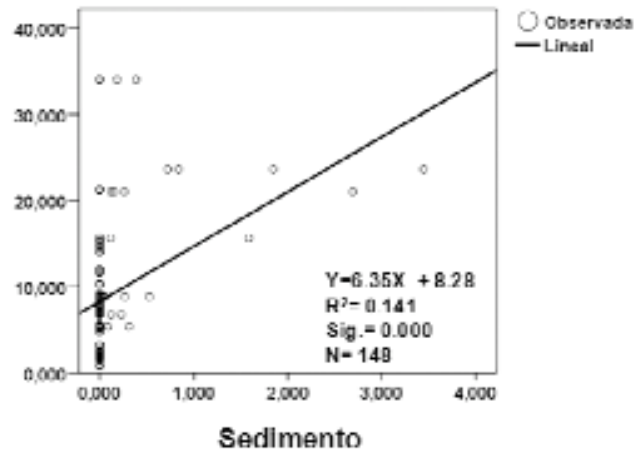

Figura 3. Representación de los modelos lineales para la generación de escorrentía y la producción de sedimento frente al volumen de precipitación y la $\mathrm{I}_{30^{\prime}}$ respectivamente.

Figure 3. Linear model graphs for runoff generation and sediment production regarding rain volume and $I_{30}$, respectively.

Tabla 6. Valores medios de los parámetros de los episodios de lluvia seleccionados y de humedad del suelo en función del tipo de respuesta de la humedad del suelo

Table 6. Average values of the rains and soil moisture parameters of the selected rain events according to the soil moisture response.

\begin{tabular}{|c|c|c|c|}
\hline & \multicolumn{3}{|c|}{ Tipo de curva de humedad del suelo } \\
\hline & Tipo I & Tipo II & Tipo III \\
\hline Número de episodios & 22 & 10 & 9 \\
\hline Precipitación & 13.2 & 35.1 & 6.0 \\
\hline$(\mathrm{mm})$ & $(1.4) \mathrm{b}$ & (6.3) a & $(0.8) \mathbf{c}$ \\
\hline $\mathrm{I}_{30}\left(\mathrm{~mm} \mathrm{~h}^{-1}\right)$ & 8.6 & 12.6 & 2.9( \\
\hline & (1.3) a & (2.6) a & $0.4) \mathbf{b}$ \\
\hline Duración & 606 & 704 & 398 \\
\hline (minutos) & (107) a & (106) a & (101) a \\
\hline $\mathrm{HS}_{a n t}(\%)$ & 11.7 & 13.5 & 10.9 \\
\hline & $(0.8) \mathrm{a}$ & (1.4) a & (1.5) a \\
\hline $\mathrm{HS}_{\text {maxx }}(\%)$ & $\begin{array}{c}15.4 \\
(0.8) \mathbf{b}\end{array}$ & $\begin{array}{c}29.7 \\
(1.6) \mathrm{a}\end{array}$ & $\begin{array}{c}11.4 \\
(1.5) \mathrm{c}\end{array}$ \\
\hline $\mathrm{HS}_{a n t} / \mathrm{HS}_{\text {máx }}$ & $\begin{array}{c}0.75 \\
(0.03) \mathbf{b}\end{array}$ & $\begin{array}{c}0.46 \\
(0.04) \mathbf{a}\end{array}$ & $\begin{array}{c}0.96 \\
(0.01) \mathbf{c}\end{array}$ \\
\hline $\begin{array}{l}\text { Descripción del tipo } \\
\text { de curva-respuesta }\end{array}$ & $\begin{array}{l}\text { Respuesta } \pm \text { rápida } \\
\text { y decrecimiento suave }\end{array}$ & $\begin{array}{l}\text { Respuesta rápida y } \\
\text { decrecimiento agudo }\end{array}$ & Respuesta baja \\
\hline
\end{tabular}

Error típico entre paréntesis. Valores medios con distinta letra indican diferencias significativas $(\mathrm{P} \leq 0.05)$.

44 (Pirineos, 2010, Vol. 165, 29-53, ISSN 0373-2568, eISSN: 1988-4281, doi: 10.3989/Pirineos.2010.165002) 
máxima del suelo $\left(\mathrm{HS}_{\text {mar }}\right)$ y la relación entre humedad antecedente y humedad máxima $\left(\mathrm{HS}_{a n} / \mathrm{HS}_{\max }\right)$, son las que diferencian claramente los tres tipos de curvas-respuesta (Figura 4):

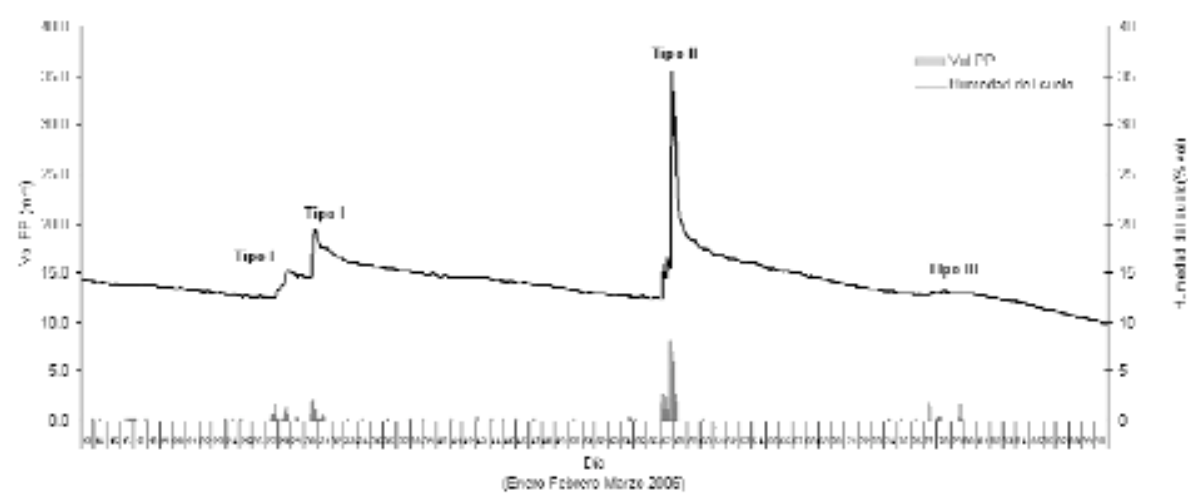

Figura 4. Diferentes tipos de curva-respuesta de la humedad del suelo en la Estación de PortaCoeli.

Figure 4. Different types of soil moisture curve-response observed in the Porta-Coeli Expeimental Station.

Tipo I: Se han identificado un total de 22 episodios, de los cuales 13 se registraron en primavera y 8 entre otoño e invierno. Se caracterizan por un claro incremento de la humedad del suelo al inicio de la precipitación y un decrecimiento posterior suave y prologando, que denota un almacenamiento significativo de agua en el suelo. Cuando la diferencia entre la $\mathrm{HS}_{\text {máx }}$ y la $\mathrm{HS}_{\text {ant }}$ es inferior a 3 unidades $(n=11)$, el incremento de humedad se produce al cabo de unas horas del inicio de la precipitación. Sin embargo, cuando la diferencia entre la $\mathrm{HS}_{\text {maxx }}$ y la $\mathrm{HS}_{\text {ant }}$ es superior a 3.4 unidades $(\mathrm{n}=11)$, se observa una respuesta rápida en el aumento de la humedad del suelo. En ambos casos, $\mathrm{HS}_{\text {max }} \mathrm{y} \mathrm{HS}_{a n t^{\prime}}$ presentan una relación lineal significativa (Figura 6A). Los episodios de lluvia que generan este tipo de respuesta se caracterizan por presentar volúmenes de precipitación inferiores a $25 \mathrm{~mm}$ (valor medio 13.2 $\mathrm{mm}$ ), cuyo rango de variación de $\mathrm{I}_{30}$ es muy amplio (entre 0.8 y $23.6 \mathrm{~mm} \mathrm{~h}^{-1}$ ). El valor medio de la relación $\mathrm{HS}_{a n t} / \mathrm{HS}_{\max }$ es de 0.75 , lo que indica que estos episodios de lluvia producen un almacenamiento de agua en el suelo, aunque también producen escorrentía.

Tipo II: Se han identificado un total de 10 episodios, la mayoría de los cuales se registraron en primavera. Se caracterizan por un brusco incremento de la humedad edáfica durante la primera hora desde el inicio de la 

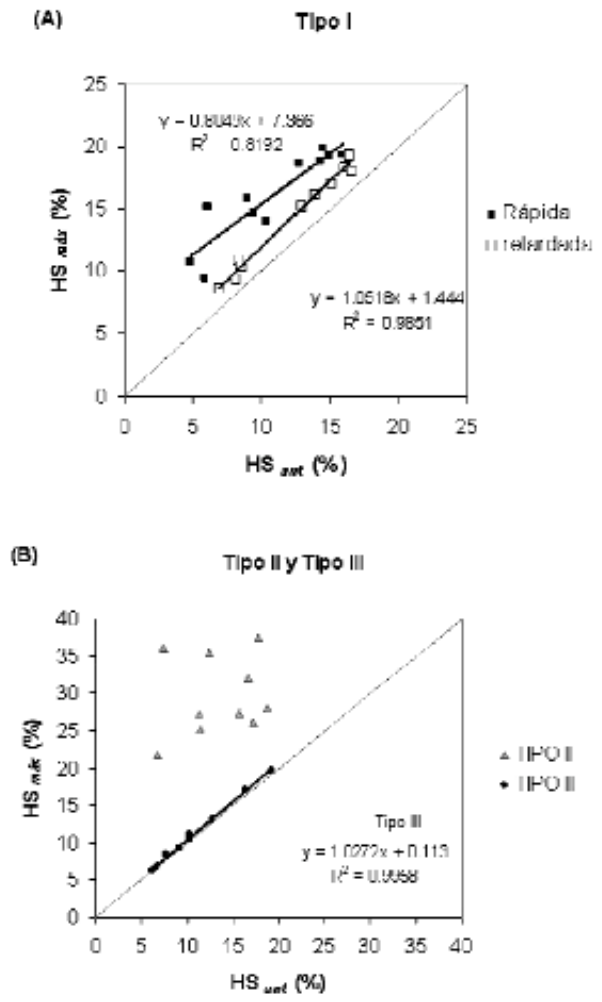

Figure 5. Relaciones entre la humedad del suelo antecedente $\left(\mathrm{HS}_{\text {ant }}\right)$ y la máxima $\left(\mathrm{HS}_{\text {máx }}\right)$ para cada tipo de curva-respuesta.

Figure 5. Correlations between background soil moisture $\left(H S_{\text {ant }}\right)$ and maximum $H S_{\text {ant }}$ for each type of curve-response.

precipitación y un descenso rápido y agudo cuando ésta cesa, lo que denota una recarga inmediata y una rápida recesión en el contenido de humedad. Los episodios de lluvia que generan este tipo de curva presentan volúmenes de precipitación superiores a $20 \mathrm{~mm}$ (valor medio $35 \mathrm{~mm}$ ) y una variación de $\mathrm{I}_{30}$ entre 3.6 y $34 \mathrm{~mm} \mathrm{~h}^{-1}$, por lo que son precipitaciones más cuantiosas y agresivas que las que caracterizaban al Tipo I. Además, este tipo de respuesta se produce cuando la $\mathrm{HS}_{a n t}$ es generalmente superior a $10 \%$, por lo que habitualmente van precedidos de episodios de lluvia con pocos días de antelación. El valor medio de la relación $\mathrm{HS}_{a n} / \mathrm{HS}_{\max }$ es de 0.46 , lo que indica la existencia de grandes diferencias entre la humedad antecedente y la máxima, pero en estos casos, no existe una buena correlación entre ambas 
(Figura 6B). En este tipo de respuesta se identifican los máximos valores de humedad del suelo (37.4\%) de todo el periodo de estudio.

Finalmente, la curva-respuesta Tipo III (Figura 5) se ha identificado en 9 episodios, la mayoría de los cuales se registran en el periodo otoño-invierno. Las variaciones del contenido de humedad son muy bajas, a pesar de que estos episodios han generado escorrentía, aunque su producción no excede los $0.05 \mathrm{~L} \mathrm{~m}^{-2}$. Esta respuesta se relaciona con las precipitaciones de volumen inferior a $10 \mathrm{~mm}$ y baja agresividad $\left(\mathrm{I}_{30} \leq 5 \mathrm{~mm} \mathrm{~h}^{-1}\right)$. En estos casos la relación $\mathrm{HS}_{a n t} / \mathrm{HS}_{\max }$ es de 0.96 , lo que denota las escasa variación respecto a la $\mathrm{HS}_{a n t^{\prime}} \mathrm{y}$ además el contenido de humedad se mantiene prácticamente constante durante varios días sucesivos.

LATRON \& GALLART (2008), también establecen una clasificación de diferentes tipos de respuesta hidrológica a escala de cuenca, que en su caso está muy relacionada con la estacionalidad y características de las precipitaciones en el Pirineo, con la dinámica del potencial hídrico y de la capa freática, y con el grado de saturación del suelo. KIM \& KIM (2007) al estudiar las variaciones temporales correspondientes a tres episodios de lluvia consecutivos, también sobre escala horaria a lo largo de una ladera, obtienen que en la parte alta se produce una respuesta similar a las curvas Tipo II de nuestro estudio, con un aumento brusco y una rápida recesión. Sin embargo, en la parte central e inferior de la ladera, describen una sucesión de curvas con ligeros aumentos y decrecimiento prolongado (Tipo I). En nuestro caso, el sensor de humedad está situado en la parte baja de las parcelas, y cuando se analizan las series temporales correspondientes a 2006-2008, se obtienen diferentes tipos de respuesta, relacionados principalmente con el volumen de precipitación de los episodios y con la relación $\mathrm{HS}_{a n t} / \mathrm{HS}_{\max }$.

De hecho, la relaciones entre las diferentes variables que caracterizan a las precipitaciones, la humedad del suelo y la producción de escorrentía (Tablas 4 y 7), muestran que el volumen y la intensidad de las lluvias presentan coeficientes rho de Spearman significativos y positivos con la producción de escorrentía en las diferentes parcelas, y coeficientes significativos y negativos con la relación $\mathrm{HS}_{a n t} / \mathrm{HS}_{\max }$. Mientras que la humedad antecedente y la humedad máxima presentan coeficientes de correlación no significativos o bajos con la producción de escorrentía, la relación $\mathrm{HS}_{a n t} / \mathrm{HS}_{\max }$ muestra coeficientes significativos y negativos con esta variable en cada una de las parcelas. La generación de escorrentía está en función de la relación $\mathrm{HS}_{a n t} / \mathrm{HS}_{\max } \mathrm{y}$ del volumen de lluvia, de modo que cuando esta relación presenta valores próximos a 1, debido a que $\mathrm{HS}_{a n t}$ es alta, volúmenes de lluvia de $2 \mathrm{~mm}$ producen escorrentía. Sin embargo, cuando la relación $\mathrm{HS}_{a n t} / \mathrm{HS}_{\max }$ es menor de 0.5 , se necesitan volúmenes de lluvia de $22 \mathrm{~mm}$ para producir escorrentía. 
Tabla 7. Coeficientes de correlación rho de Spearman entre las variables relacionadas con las características de las precipitaciones (1), la humedad del suelo y la producción de escorrentía en los tratamientos de la Estación Experimental de Porta-Coeli.

Table 7. Rho Spearman correlation coefficients between variables related to the rains characteristics (1), soil moisture and runoff generation in the treatments of the Porta-Coeli Experimental Station.

\begin{tabular}{|c|c|c|c|c|c|c|c|c|}
\hline & Volumen PP & $I_{30}$ & $H S_{\text {ant }}$ & $H S_{\text {máx }}$ & $\begin{array}{l}H S_{\text {ant }} / \\
H S_{\text {máx }}\end{array}$ & $R Y-T 1$ & $R Y-T 2$ & $R Y-T 3$ \\
\hline Volumen PP & - & & & & & & & \\
\hline $\mathrm{I}_{30}$ & 0.660 ** & - & & & & & & \\
\hline HS ant & n.s. & n.s. & - & & & & & \\
\hline HS ${ }_{\text {max }}$ & $0.558^{* *}$ & n.s & $0.664^{* *}$ & - & & & & \\
\hline HS $\operatorname{maxt}_{\text {max }} / \mathrm{HS}$ & $-0.834^{* *}$ & $-0.758^{* *}$ & n.s. & $-0.529^{* *}$ & - & & & \\
\hline RY-T1 (2) & $0.703^{* *}$ & $0.603^{* *}$ & n.s. & $0.476^{*}$ & $\begin{array}{c}- \\
0.753^{* *}\end{array}$ & - & & \\
\hline RY-T2 & $0.592^{* *}$ & $0.637^{\star *}$ & n.s. & $0.468^{*}$ & $\begin{array}{c}- \\
0.675^{* *}\end{array}$ & $0.695^{\star *}$ & - & \\
\hline RY-T3 & $0.609^{* *}$ & $0.554^{* *}$ & n.s. & $0.485^{*}$ & $\begin{array}{c}- \\
0.718^{* *}\end{array}$ & $0.823^{\star *}$ & $\begin{array}{c}0.625^{* *} \\
* *\end{array}$ & - \\
\hline RY-T4 & $0.709^{* *}$ & $0.692^{* *}$ & n.s. & 0.440 & $\begin{array}{c}- \\
0.815^{\star *}\end{array}$ & $0.925^{\star *}$ & $0.675^{\star *}$ & $0.896^{* *}$ \\
\hline
\end{tabular}

(1) no se incluye la duración del episodio porque los coeficientes son no significativos.

(2) RY: producción de escorrentía $\left(\mathrm{L} \mathrm{m}^{-2}\right)$ en los distintos tratamientos (T1, T2, T3 y T4).

${ }^{*},{ }^{* *}$ : la correlación es significativa al nivel 0.05 y 0.01 respectivamente.

\subsection{Producción de sedimento}

La pérdida de suelo observada en los distintos tratamientos durante 20062008 ha sido mínima, en gran parte debido a la moderada agresividad de las precipitaciones, las características hidrológicas que favorecían una infiltración eficaz y una ostensible mejora en las condiciones estructurales del suelo. De los 321 episodios de lluvia ocurridos durante el estudio sólo en 7 se apreció producción de sedimento, y en algún caso no en todos los tratamientos. Esto supone que solo un 2.2\% del total de lluvias generaron datos. Este reducido número de datos ha dificultado de alguna manera el análisis estadístico de los mismos.

En general, las pérdidas de suelo medidas en estos 7 episodios han sido muy reducidas (Tabla 3), con un máximo de $3.4 \mathrm{~g} \mathrm{~m}^{-2}$ correspondiente al tratamiento $\mathrm{T} 4$ en 2008, y con una media de $0.10 \mathrm{~g} \mathrm{~m}^{-2}$ para todo el período. Estos valores han sido inferiores a todos los encontrados en la bibliografía consultada para condiciones ambientales y de tratamientos similares. No obstante, los valores presentan diferencias claras entre los tratamientos 
aunque no sean estadísticamente significativas. Así, los tratamientos que han sufrido impactos de mayor importancia, T1 y T4, presentan mayores valores que T2 y T3. Esto se ve reflejado en el año 2008 donde sólo hubo un episodio de lluvia en el que se apreció producción de sedimento y solo en T1 y T4, no en los demás tratamientos. Como ocurría respecto a la generación de escorrentía, en todos los casos T4 (parcela que se mantuvo sin vegetación hasta el 2000) dio mayores niveles de producción de sedimento que T1 (parcela que sufrió la quema experimental).

Respecto a los valores de arrastre de suelo el perfil es muy similar en su comportamiento, presentando valores bajos en todos los casos (Tabla 3), con una media global para todo el período estudiado de $0.25 \mathrm{~g} \mathrm{~L}^{-1}$. El valor máximo alcanzado también se dio en $\mathrm{T} 4$ con $7.62 \mathrm{~g} \mathrm{~L}^{-1}$.

En el análisis de los datos referentes a estos dos parámetros (Tablas 4 y 5) se ha observado que en ambos casos es la intensidad de la lluvia $\left(\mathrm{I}_{30}\right)$ el factor determinante. Sólo en el caso de T4 para la producción de sedimento aparece como factor de mayor influencia la escorrentía generada, junto con la $\mathrm{I}_{30}$. Este dato podría indicar, como ocurría con la producción de escorrentía, una menor recuperación en las condiciones hidrológicas y edáficas de T4 respecto a los demás tratamientos.

Estas tendencias se han observado también en el ajuste a diferentes modelos, donde se observó que en todos los casos la producción de sedimento y el arrastre se representan mejor en un modelo lineal frente a la intensidad de la lluvia (Figura 4). En todos los tratamientos, también podrían ajustarse positivamente a un modelo cuadrático.

En el caso de T4, la producción de sedimento también daría un modelo lineal con la escorrentía generada.

\section{Conclusiones}

La gran variabilidad climática interanual, con grandes fluctuaciones en la distribución y características de las precipitaciones, es un factor determinante en la respuesta hidrológica de los suelos estudiados en sus distintos tratamientos. La recuperación de la cobertura vegetal y mejora en las características del suelo desde el año 2000 ha incidido en una mejora general en el comportamiento hidrológico de los distintos tratamientos. Esto se ha traducido en unos bajos niveles de generación de escorrentía y, sobre todo, de producción de sedimento.

Pero, independientemente de esta recuperación, las diferencias entre tratamientos se mantienen. Se ha observado una mejora patente en los tratamientos en los que se emplearon medidas de reforestación con especies 
arbustivas para el control de erosión (T2 y T3). Mientras que el tratamiento que se mantuvo sin vegetación durante 10 años (T4) es el que presenta los valores más altos tanto en la generación de escorrentía como en la pérdida de suelo, seguido por el tratamiento que sufrió el impacto del fuego (T1).

La intensidad de la precipitación $\left(\mathrm{I}_{30}\right)$ es el factor más importante respecto tanto a la generación de escorrentía como a la pérdida de suelo y al arrastre de este en todos los tratamientos. Sólo en caso del tratamiento mantenido sin vegetación es el volumen de lluvia el factor de mayor peso, mas incluso que la escorrentía.

Se han identificado tres tipos de curvas-respuesta de la humedad del suelo. Las respuestas dominantes son las de Tipo I (respuesta algo retardada y decrecimiento prologado y suave) y Tipo II (respuesta rápida y decrecimiento agudo). Tanto el contenido volumétrico de humedad máximo como la relación $\mathrm{HS}_{a n t} / \mathrm{HS}_{\text {máx }}$ influyen directamente en el volumen de escorrentía generado en cada episodio de lluvia. En este caso, la combinación de pocos puntos de medida espaciales de la humedad edáfica pero con alta resolución temporal y con medidas continúas, contribuye a la obtención de información sobre la generación de escorrentía en las parcelas experimentales.

En ambientes mediterráneos, con fuertes variaciones climáticas y una reducida precipitación, la pérdida o degradación de la cubierta vegetal y el impacto de los incendios forestales inciden directamente en la alteración de las características hidrológicas de los suelos y en su respuesta a los procesos erosivos.

\section{Agradecimientos}

Este trabajo ha sido realizado en el ámbito del proyecto CONSOLIDER: PROBASE, CGL2006-11619/HID, financiado por el Ministerio de Ciencia y Tecnología, dentro de la Red RESEL del Ministerio de Medio Ambiente y en terrenos cedidos por la Generalitat Valenciana para la construcción de la Estación Experimental.

\section{Referencias}

ANDREU, V., RUBIO, J.L. \& CERNI, R., 1998. Effects of Mediterranean shrub cover on water erosion (Valencia, Spain). Journal of Soil and Water Conservation, 53 (2): 112-120.

ANDREU, V., IMESON, A.C. \& RUBIO, J.L., 2001. Temporal changes in soil 
aggregates and water erosion after a wildfire in a Mediterranean pine forest. Catena, 44: 69-84.

BAKKER, M.M., GOVERS, G., VAN DOORN, A., QUETIER, F., CHOUVARDAS, D. \& ROUNSEVELL, M., 2008. The response of soil erosion and sediment export to land-use change in four areas of Europe: The importance of landscape patterns. Geomorphology 98: 213-226.

BEGUERIA, S., LÓPEZ-MORENO, J., GÓMEZ-VILLAR, A., RUBIO, V. LANA-RENAULT, N. \& GARCÍA-RUIZ, J., 2006. Fluvial adjustments to soil erosion and plant cover changes in the Central Spanish Pyrenees. Geografiska Annaler, Series A: Physical Geography 88: 177-186.

BOIX-FAYOS, C., DE VENTE, J., MARTINEZ-MENA, M., BARBERÁ, G.G. \& CASTILLO, V., 2008. The impact of land use change and chef-dams on catchment sediment yield. Hydrological Processes, 22: 4922-4935.

CHIN, D.A, 2006. Water-Resources Engineering (2 ${ }^{\text {nd }}$ Edition). Prentice-Hall, 962 pp. New York.

CAMPO, J., ANDREU, V., GIMENO-GARCÍA, E., GONZALEZ, O. \& RUBIO, J.L., 2006. Occurrence of soil erosion after repeated experimental fires in a Mediterranean environment. Geomorphology, 82: 376-387.

CORBITT, R. A., 1999. Standard Handbook of Environmental Engineering. McGraw-Hill, 2 ed. 1152 pp., New York.

CHRISTENSEN, J.H., HEWITSON, B., BUSUIOC, A., CHEN, A., GAO, X., HELD, I., JONES, R., KOLLI, R.K., KWON, W.-T., LAPRISE, R., MAGAÑA-RUEDA, V., MEARNS, L., MENÉNDEZ, C.G., RÄISÄNEN, J., RINKE, A., SARR, A. \& WHETTON, P., 2007. Chapter 11: Regional Climate Projections. En: Climate Change 2007: The Physical Science Basis. Contribution of Working Group I to the Fourth Assessment Report of the Intergovernmental Panel on Climate Change. (S. Solomon, D. Qin, M. Manning, Z. Chen, M. Marquis, K.B. Averyt, M. Tignor y H.L. Miller, Eds). Cambridge University Press, pp. 847-940, Cambridge.

DOERR, S.H., SHAKESBY, R.A., BLAKE, W.H., CHAFER, C.J., HUMPHREYS, G.S. \& WALLBRIK, P.J., 2006. Effects of differing wildfire severities on soil wettability and implications for hydrologyc response. Journal of Hydrology, 319: 295-311.

EUROPEAN COMMISSION, 2003. Forest fires in Europe: 2002 fire campaign. Official Publication of the European Communities. SPI03.83EN, European Communities/JRC, 89 pp., Luxembourg.

EUROPEAN ENVIRONMENT AGENCY - EEA, 2005. The European environment - State and outlook 2005. Part A: Integrated Assessment. Office for Official Publications of the European Communities, 227 pp., Copenhaguen. 
FAULKNER, H., RUIZ, J., ZUKOWSKYJ, P \& DOWNWARD, S., 2003. Erosion risk associated with rapid and extensive agricultural clearances on dispersive materials in southeast Spain. Environmental Science \& Policy 6(1): 115-127.

GOVERS, G., VAN OOST, K. \& POESEN, J., 2006. Responses of a semi-arid landscape to human disturbance: A simulation study of the interaction between rock fragment cover, soil erosion and land use change. Geoderma 133: 19-31.

INTERGOVERNMENTAL PANEL ON CLIMATE CHANGE - IPCC, 2007. Summary for Policymakers. In M.L. Parry, O.F. Canziani, J.P. Palutikof, P.J. van der Linden and C.E. Hanson [Eds.], Climate Change 2007: Impacts, Adaptation and Vulnerability. Contribution of Working Group II to the Fourth Assessment Report of the Intergovernmental Panel on Climate Change. Cambridge University Press, pp. 7-22, Cambridge.

JOEL, A., MESSING, I., SEGUEL, O. \& CASANOVA, M., 2002. Measurement of surface water runoff from plots of two different sizes. Hydrological Processes, 16: 1467-1478.

JOHANSEN, M.P., HAKONSON, T.E. \& BRESHEARS, D.D., 2001. Post-fire runoff and erosion from rainfall simulation: contrasting forest with shrublands and grasslands. Hydrological Processes, 15: 2953-2965.

KIM, S. \& KIM, H., 2007. Stochastic análisis of soil moisture to understand spatial and temporal varaitions of soil wetness at a steep hildside. Journal of Hydrology, 341: 1-11.

KOULOURI, M. \& GIOURGA, C., 2007. Land abandonment and slope gradient as key factors of soil erosion in Mediterranean terraced lands. Catena, 69: 274-281.

LASANTA-MARTINEZ, T., VICENTE-SERRANO, S. \& CUADRAT-PRATS, J.M., 2005. Mountain Mediterranean landscape evolution caused by the abandonment of tradicional primary activities: a study of the Spanish Central Pyrenees. Applied Geography, 25: 47-65.

LATRON, J. \& GALLART, F., 2008. Runoff generation processes in a small Mediterranean research catchment (Vallcebre, Eastern Pyrenees). Journal of Hydrology, 358: 206-220.

LLOVET, J., RUIZ-VALERA, M., JOSA, R. \& VALLEJO, R., 2009. Soil response to fire in Mediterranean forest landscapes in relation to the previous stage of land abandonment. Internacional Journal of Wildland Fire, 18: 222-232.

MICKELSON, S.K., BOYD, P., BAKER, J.L. \& AHMED, S.I., 2001. Tillage and herbicide incorporation effects on residue cover, runoff, erosion, and herbicide loss. Soil \& Tillage Research, 60: 55-66.

PARDINI, G., GISPERT, M. \& DUNJO, G., 2003. Runoff erosion and nutrient 
RESPUESTA HIDROLÓGICA Y EROSIVA DE UN SUELO FORESTAL MEDITERRÁNEO...

depletion in five Mediterranean soils of NE Spain under different land use. Science of the Total Environment, 309 (1-3): 213-224.

PARDINI, G., GISPERT, M. \& DUNJO, G,. 2004. Relative influence of wildfire on soil properties and erosion processes in different Mediterranean environments in NE Spain. Science of the Total Environmen, 328: 237-246.

PIERSON, F.B., ROBICHAUD, P.R., MOFFET, C.A., SPAETH, K.E., HARDEGREE, S.P., CLARK, P.E. \& WILLIAMS, C.J., 2008. Fire effects on rangeland hydrology and erosion in a steep sagebrush-dominated landscape. Hydrological Processes, 22: 2916-2929.

PROSSER, I.P. \& WILLIAMS, L., 1998. The effect of wildfire in runoff and erosion in native Eucalyptus forest. Hydrologycal Processes, 12: 251-265.

RUBIO, J.L., FORTEZA, J., ANDREU, V. \& CERNI, R., 1997. Soil profile characteristics influencing runoff and soil erosion after forest fire: a case study (Valencia, Spain). Soil Technology, 11: 67-78.

SHAKESBY, R.A. \& DOERR, S.H., 2006. Wildfire as a hydrological and geomorphological agent. Earth-Science Reviews, 74: 269-307. 